\title{
Overexpression of fasciculation and elongation protein $\zeta-1$ (FEZ1) induces a post-entry block to retroviruses in cultured cells
}

\author{
Mojgan H. Naghavi, ${ }^{1}$ Theodora Hatziioannou, ${ }^{1,2}$ Guangxia Gao, ${ }^{1,3}$ and Stephen P. Goff ${ }^{1,4}$ \\ ${ }^{1}$ Department of Biochemistry and Molecular Biophysics, Howard Hughes Medical Institute, Columbia University, College of \\ Physicians and Surgeons, New York, New York 10032, USA; ${ }^{2}$ Aaron Diamond AIDS Research Center, New York, New York \\ 10016, USA; ${ }^{3}$ Institute of Microbiology, Chinese Academy of Sciences, Beijing 100080, China
}

Two mutant Rat2 fibroblast cell lines, R3-2 and R4-7, have been previously isolated by a selection for retrovirus resistance. We have now further analyzed the basis of the block to retroviral infection in the R3-2 line. Using Affymetrix GeneChip analysis, several genes were identified as differentially expressed in the mutant R3-2 line compared with the wild-type cells. One of the candidate gene products, FEZ1 (fasciculation and elongation protein $\zeta-1)$, a protein kinase $\mathrm{C}(\mathrm{PKC}) \zeta$-interacting protein homologous to the Caenorhabditis elegans synaptic transport protein UNC-76, was found to be up-regulated $>30$-fold in the resistant R3-2 line. FEZ1 overexpression in Rat 2 cells conferred a potent resistance to infection by genetically marked retroviruses, and the degree of retroviral resistance in both Rat 2 fibroblasts and $293 \mathrm{~T}$ cells tightly correlated with the expression level of FEZ1 transcripts. FEZ1-overexpressing Rat2 cells showed a similar phenotype to that of the mutant R3-2 line: Infection resulted in normal viral DNA synthesis but a reduction in the formation of circular DNA, indicating a block after reverse transcription but before nuclear entry. Partial knockdown of FEZ1 expression in R3-2 by RNA interference (RNAi) significantly reduced the resistance of this line to infection. Thus, our data suggest that FEZ1 overexpression is sufficient to explain the resistant phenotype of R3-2 cells and identify FEZ1 as a new gene capable of causing retrovirus resistance.

[Keywords: Retroviruses; viral block; cellular factors; FEZ1]

Supplemental material is available at http://www.genesdev.org.

Received December 15, 2004; revised version accepted March 24, 2005.

The coexistence of vertebrates and retroviruses over millions of years has resulted in the evolution of multiple defense mechanisms to inhibit viral replication in vertebrate cells (for recent reviews, see Bieniasz 2003; Goff 2004). Several genes have recently been identified that induce intracellular blocks to retrovirus infection. These are exemplified by the mouse gene Fv1 (Friend-virus-susceptibility-1), which prevents retroviral infection after reverse transcription but before nuclear entry and integration of the provirus (Pincus et al. 1971); the APOBEC3G enzyme, which is incorporated into virion particles and induces destructive deamination of the viral cDNA as it is synthesized (Harris et al. 2003); the Zinc finger Antiviral Protein (Zap), which inhibits the accumulation of many RNAs to prevent viral replication (Gao et al. 2002); and the tripartite interaction motif $5 \alpha$

${ }^{4}$ Corresponding author.

E-MAIL goff@cancercenter.columbia.edu; FAX (212) 305-5106. Article and publication are at http://www.genesdev.org/cgi/doi/10.1101/ gad.1290005
(TRIM5 $\alpha$ ) protein, which somehow blocks incoming virus at an early post-entry step before reverse transcription (Stremlau et al. 2004). The retrovirus resistance factors named Ref1 (restriction factor-1) and Lv1 (Lentivirus susceptibility-1) were recently shown to be the speciesspecific variants of TRIM5 $\alpha$ (Hatziioannou et al. 2004; Keckesova et al. 2004).

This growing list of intracellular defenses against viral attack suggests that more novel antiviral genes are yet to be identified. In an attempt to identify more such genes, virus-resistant cell mutants were isolated after chemical mutagenesis and direct selection for resistance to retrovirus infection. Two such resistant lines, R4-7 and R3-2, isolated from a parental fibroblast line, Rat2 cells, have been characterized in detail (Gao and Goff 1999). Mutant line R4-7 exhibited $\sim 100$-fold resistance to infection by genetically marked Moloney murine leukemia viruses (MLVs), and was resistant to pseudotyped viruses utilizing the amphotropic or ecotropic envelope or even the vesicular stomatitis virus (VSV-G) envelope protein. Infection of R4-7 was blocked before the initiation of re- 
verse transcription. Recently, two cDNA constructs that each could restore virus sensitivity to the R4-7 mutant line were isolated (Gao and Goff 2004). Mutant line R3-2 exhibited a nearly 1000 -fold resistance to infection by $M L V$, also independent of the envelope mediating entry. Infection of this line resulted in the normal levels of the linear viral DNA synthesis by reverse transcription but excluded the formation of circular viral DNAs and integrated proviruses from the nucleus. The viral DNA was apparently trapped by an unknown mechanism in the cytoplasm in a form that could not be readily extracted under conditions that allowed the DNA recovery from the wild-type-infected cells. Both mutant lines were also resistant to infection by human immunodeficiency virus type 1 (HIV-1) pseudotypes.

In this study we have further analyzed the genetic block in the mutant R3-2 line. We identified several highly expressed genes as candidates to explain the resistance phenotype of this line. Among these gene products, FEZ1 (fasciculation and elongation protein $\zeta-1$ ), a mammalian homolog of the Caenorhabditis elegans UNC-76 protein, was up-regulated $>30$-fold in the resistant R3-2 line. A direct target of protein kinase C (PKC) $\zeta$ dependent signaling (Kuroda et al. 1999) and a kinesin associated-protein essential for synaptic vesicle transport (Gindhart et al. 2003), FEZ1/UNC-76 became a primary candidate to explain the exclusion of viral DNA from the nucleus in the R3-2 line. We here provide support for the hypothesis that FEZ1 overexpression contributes to the resistant phenotype of R3-2 cells.

\section{Results \\ Identification of differentially expressed genes in the R3-2 cell line}

To identify genes that could explain the resistant phenotype of the R3-2 line, we first attempted to identify differentially expressed genes. To establish gene expression profiles of the wild-type and the mutant R3-2 line, labeled cRNA was generated and hybridized to rat GeneChips that monitor expression of 8800 rat transcripts. Pairwise comparison analyses of the mutant versus the wild-type genes were carried out by using the Affymetrix Microarray Suite 5.0 software. The comparisons were then sorted by ascending or descending fold change (FC) ratios to obtain genes that were turned up or down, respectively, in the mutant R3-2 line compared to that of the wild-type Rat 2 cells (see Material and Methods). The array data demonstrated that most genes (87\%) did not show expression changes according to our criteria for significance (average change eightfold or more and detection $p$-value $<0.05$ ) (data not shown). After elimination of these genes, 1150 transcripts were identified as potential differentially expressed genes. Among them, only 118 genes, which accounted for $\sim 1.3 \%$ of the total genes examined, showed consistent FC ratios obtained from five or more pairwise comparisons. Thirty-nine of these affected genes with the greatest average FC, between 695- and 17-fold, are presented in Table 1. The data also
Table 1. Differentially expressed genes in the mutant R3-2 line

\begin{tabular}{|c|c|c|}
\hline Gene & Accession no. & $\begin{array}{c}\text { Fold } \\
\text { change }\end{array}$ \\
\hline RMCP-10 & U67913 & 695.1 \\
\hline RMCP-8 & U67911 & 655.8 \\
\hline${ }^{a}$ RANTES & AI009658 & 553.4 \\
\hline${ }^{a}$ Phospholipase A2 & X51529 & 172.1 \\
\hline${ }^{a}$ Unknown & AI639162 & 162.1 \\
\hline${ }^{a} 53-\mathrm{kDa}$ polypeptide & X02601 & 157.9 \\
\hline FEZ1 & U63740 & 140.7 \\
\hline${ }^{a} \mathrm{GST}-\mathrm{Yc}$ & K01932 & 131.1 \\
\hline${ }^{a} \mathrm{Xdh}$ & AI172247 & 97.8 \\
\hline${ }^{a} \mathrm{FXYD}$ & AA799645 & 83.7 \\
\hline GluR-K3 & $\mathrm{X} 54656$ & 83.5 \\
\hline${ }^{a}$ Isoprenylated-67 kDa & M80367 & 57.9 \\
\hline Sharp-1 & AF009329 & 53.6 \\
\hline${ }^{a}$ Dynamin & X54531 & 52.0 \\
\hline T-ALDH & J03637 & 48.8 \\
\hline Myosin & S68736 & 44.8 \\
\hline${ }^{a} \alpha$-actin & AA900769 & 43.4 \\
\hline Maspin & U58857 & 38.1 \\
\hline$\alpha$-COLIII & X70369 & 35.4 \\
\hline JAK2 & U13396 & 35.3 \\
\hline Ceruloplasmin & AA817854 & 32.7 \\
\hline MAP2 & X17682 & 32.6 \\
\hline C-FABP & S69874 & 30.8 \\
\hline VAMP-1 & M24104 & 30.3 \\
\hline CD81/TAPA-1 & AI103957 & 29.8 \\
\hline${ }^{a}$ chemokine CX3C & AF030358 & 28.6 \\
\hline $\mathrm{COX} 2$ & S67722 & 27.2 \\
\hline Maguin & AA893357 & 26.7 \\
\hline CASK & U47110 & 26.0 \\
\hline Rev-ErbA- $\beta$ & U20796 & 24.3 \\
\hline LGR4 & AF061443 & 22.8 \\
\hline PC3 & M60921 & 22.4 \\
\hline PCIP1 & AF056208 & 21.6 \\
\hline AT-BP2 & X54250 & 21.0 \\
\hline $\mathrm{Mdm} 2$ & AI639488 & 20.9 \\
\hline Gelatinase A & U65656 & 18.8 \\
\hline MEKK1 & U48596 & 18.7 \\
\hline Kid-1 & M96548 & 18.3 \\
\hline Toll & AF057025 & 17.3 \\
\hline
\end{tabular}

Fold change ratios are mean of the five or more pairwise comparisons between the mutant R3-2 line and the wild-type Rat2 cells. Genes indicated in bold text are down-regulated in R3-2. ${ }^{a}$ Genes that are up-regulated in both the mutant lines, R3-2 and R4-7.

showed that while the majority of these genes were upregulated in R3-2, only three genes (indicated in bold text in Table 1) were down-regulated in this line compared with that of the wild-type Rat 2 cells. However, the latter genes and several of the up-regulated transcripts in R3-2 line (indicated in Table 1) showed the same altered expression patterns in the other previously reported mutant line R4-7 (Gao and Goff 1999; data not shown). In contrast to R3-2, the viral block in R4-7 was shown to be before reverse transcription of the viral RNA into DNA (Gao and Goff 1999). Thus, the commonly affected genes in both of the virus-resistant mutant lines were not considered likely candidates to explain the two distinctive 
blocks in these lines but rather may reflect clonal variation present in the mutagenized cell population. The genes that were specifically affected in R3-2 were therefore selected for further analysis.

\section{Confirmation of microarray results by quantitative real-time PCR}

To confirm the changes detected by the microarray chips, quantitative RT-PCR was utilized. Those 25 genes that were most highly up-regulated in R3-2 compared with that of the wild-type Rat 2 cells were selected for confirmation. For the sequences of the primers and the PCR product sizes used in the quantitative RT-PCR, see Supplementary Table 1. The average FC ratios detected by GeneChip analysis and by RT-PCR are indicated side by side for each gene in Figure 1. In general, the direction of differential gene expression obtained by the GeneChip analysis and the RT-PCR assays was similar for all the genes tested here. For example, genes that were found to be up-regulated by the array results showed also a higher expression level detected by the RT-PCR. Despite that, the array results accurately reflected the magnitude of the changes in gene expression patterns detected by RT-PCR for only about one-third of the genes, while the remaining transcripts showed smaller changes by RT-PCR. As a control, one of the common genes (dynamin) that displayed a higher expression level in both of the mutant lines, R3-2 and R4-7, compared with that of the wild-type Rat 2 cells, was also tested. The increase in dynamin expression by the RTPCR assay was consistent with that observed in the microarray experiment for both of the mutant lines (data not shown). These data indicate that differential expression changes between the mutant R3-2 line and the wildtype Rat2 cells in the microarray data were confirmed, though the quantitative RT-PCR assays often indicated quantitatively smaller changes.

\section{Overexpression of FEZ1 blocks retrovirus infection in Rat2 cells}

Data from the microarray chip analysis and confirmed by quantitative RT-PCR revealed seven genes as candidates to explain the resistant phenotype of the mutant R3-2 line. Among these gene products, FEZ1, a mammalian homolog of the C. elegans UNC-76 protein, was up-regulated $>30$-fold in the resistant R3-2 line. A direct target of PKC $\zeta$-dependent signaling (Kuroda et al. 1999) and essential for synaptic vesicle transport (Gindhart et al. 2003), FEZ1/UNC-76 became a primary candidate to explain the exclusion of viral DNA from the nucleus in the R3-2 line (Gao and Goff 1999). To test whether overexpression of FEZ1 could block retrovirus infection, Rat 2 cells were transfected with DNAs expressing FEZ1 plus the Zeocin selectable marker, and stable drug-resistant cell lines were isolated. Seven clones of Rat2 cells stably expressing a variant myc-His-tagged FEZ1 (m-FEZ1; see below)

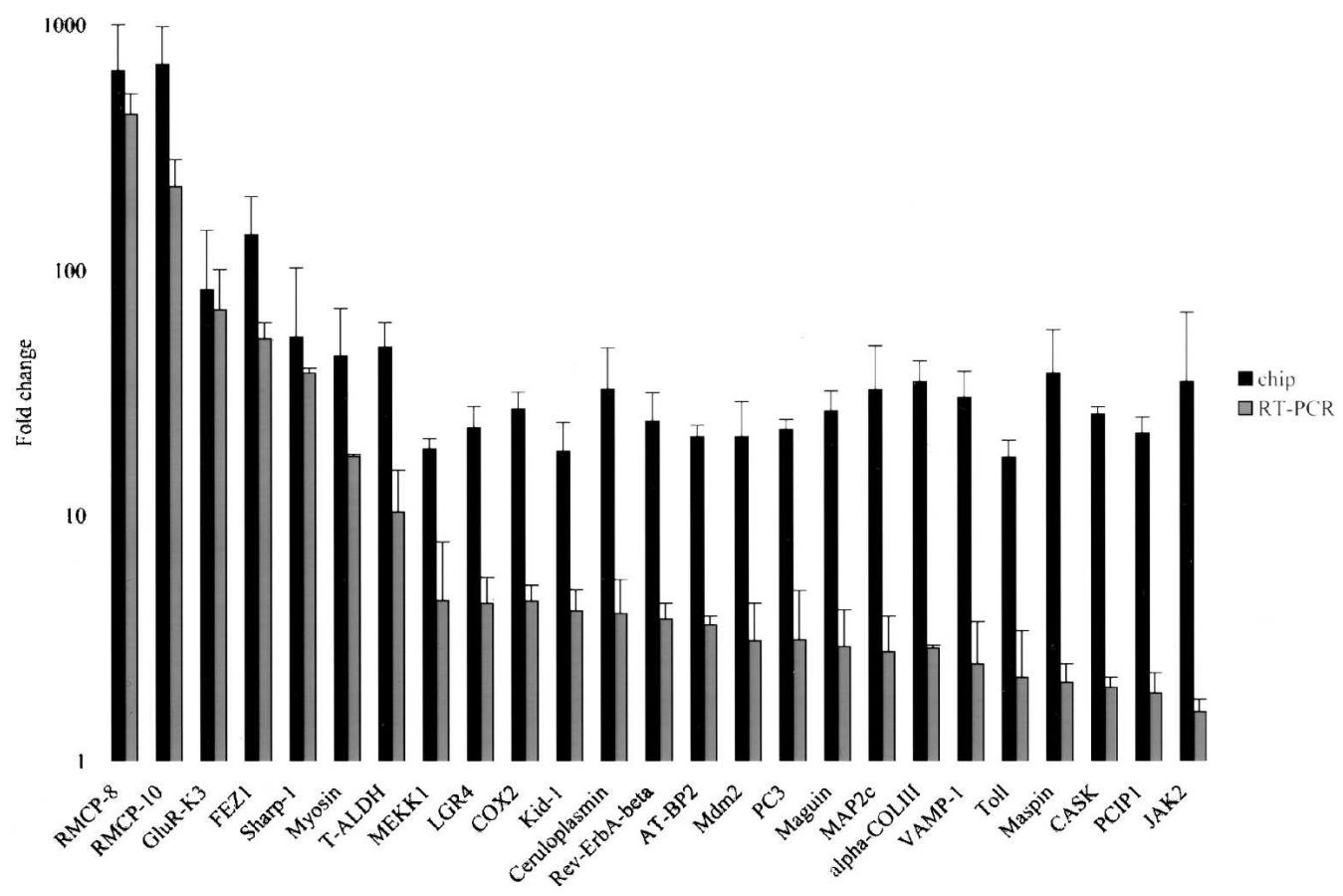

Figure 1. Microarray chip and quantitative RT-PCR data showing genes that are up-regulated in the mutant R3-2 line. FC ratios obtained from the chip analysis are mean of the five or more pairwise comparisons between the mutant R3-2 line and the wild-type Rat 2 cells. The FC ratio between the mutant line and the control wild-type cells detected in the RT-PCR analysis are median copy numbers normalized to GAPDH copy numbers for each gene. The RT-PCR results shown for each transcript are typical of those obtained in duplicate using RNA from at least two independent preparations. 
were recovered. These clones and two control Rat 2 cells containing empty vector, wild-type Rat2 cells, and the parental R3-2 mutant line were challenged by infection with retroviral preparations of MLV-based vectors carrying the Neomycin resistance marker (MLV-N-neo, MLVB-neo), and with an HIV-based vector carrying puromycin resistance. The MLV or HIV-1-infected cells were selected in medium containing G418 or puromycin, respectively. The number of transduced colonies was then counted after 8-12 d. The majority of the clones expressing FEZ1 were highly resistant to MLV-N-neo virus compared with the parental cells or cells carrying the empty vector (Fig. 2A). The clones showed different levels of resistance to the virus, with the highest $>100$-fold (mFEZ1:11) resistant as compared to cells transduced with the empty vector (pcDNA4:3). The average block to MLV-N-neo was 47-fold in the FEZ1-expressing lines, which was similar to that originally reported for R3-2 (Gao and Goff 1999). Very similar results were obtained with MLV-B-neo infection, where the FEZ1-expressing lines were 39-fold resistant compared with controls (Fig. 2B). The majority of the stable clones restricted MLV-Bneo infection to a greater extent than MLV-N-neo (Fig. 2A). Similar test of NB-tropic viruses pseudotyped with VSV-G envelope protein of a subset of these cells gave very similar results to MLV-N-neo infection (data not shown). To test whether FEZ1 overexpression was only active against MLV retroviral infection or could also inhibit recombinant HIV-1, we tested for FEZ1 activity in HIV-1-puro-infected cells. As is shown in Figure 2C, overexpression of FEZ1 also blocked HIV-1-puro in Rat2 cells. The levels of resistance detected against HIV-1puro virus were comparable to that obtained against both MLV preparations (Fig. 2A,B) in the same FEZ1-expressing lines. In conclusion, overexpression of FEZ1 was sufficient to establish significant retroviral resistance in Rat2 cells.

\section{Both the wild-type and a variant FEZ1 are functional for inhibition}

Sequence analysis of the FEZ1 cDNA isolated from R3-2 cells (m-FEZ1) revealed two nucleotide changes relative to the published sequences at nucleotides 936 and 997; one (936) was silent while the other (997) caused an amino acid change of Phe to Ile. In addition, m-FEZ1 lacked an entire codon, encoding Glu at position 98. To test for the possible origin of these differences, we sequenced multiple cDNAs from both wild-type and R3-2 cells. The changes at these nucleotides occurred at a similar frequency in both wild-type Rat2 cells and mutant R3-2 lines, with the variant allele expressed at a frequency of $\sim 20 \%$ (data not shown). Thus, both the parental line and the mutant cells are apparently polymorphic (and most likely heterozygous) at the FEZ1 locus. To verify that these changes did not alter the ability of FEZ1 to confer resistance to retroviral infection, 10 clones of Rat2 cells expressing either the wild-type or the variant Flag-tagged FEZ1, the control Rat2 cells containing empty vector, and the R3-2 line were tested in MLV-
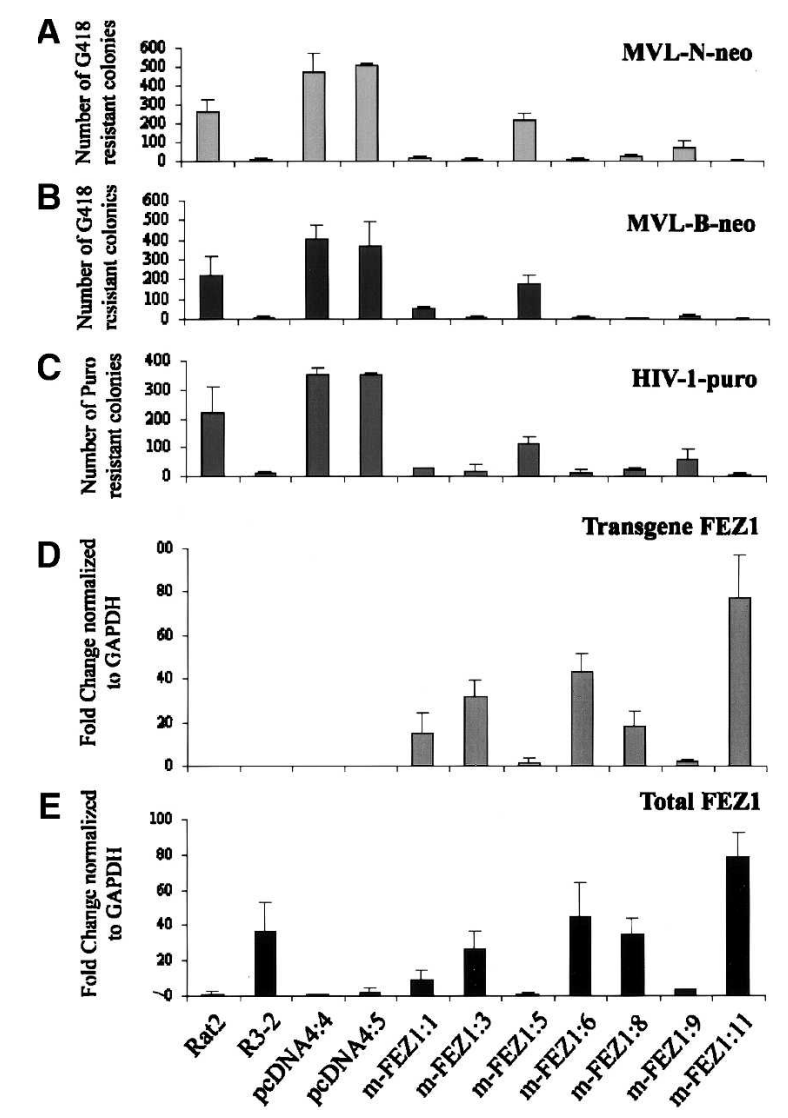

Figure 2. The pattern of expression levels of FEZ1 transcripts correlates with the levels of retroviral resistance in the various Rat2 lines. $(A-C)$ Overexpression of FEZ1 blocks retrovirus infection in Rat2 cells. Cells transduced with the empty vector pcDNA4 or the pcDNA4 vector expressing FEZ1 originated from the mutant R3-2 line, m-FEZ1, were incubated with various amounts of MLV-N-neo $(A)$, MLV-B-neo $(B)$, and HIV-1-puro $(C)$. Cells were selected in medium containing G418 (A,B; Neoviruses) or puromycin ( $C$; HIV-1) and the number of the transduced colonies were then counted after 8-12 d. The wild-type Rat2 cells and the parental R3-2 line were included as negative and positive controls, respectively. Similar results were obtained in at least three independent experiments. $(D, E)$ Quantitative RT-PCR showing the level of FEZ1 expression in Rat2 cells. Cytoplasmic RNA was prepared from the same cell lines used in the transduction assay. The ds cDNA was generated and used as template in RT-PCR assays. Primers unique to each cDNA sequences were used to distinguished the transgene FEZ1 $(D)$ from the total (endogenous and transgene) (E) FEZ1 transcripts. The FC ratio between the Rat 2 mutant lines and the control empty vector line (pcDNA4:3) are given as median copy numbers normalized to GAPDH copy numbers obtained in duplicate from two independent RNA preparations.

$\mathrm{N}$-neo and HIV-1-puro infection assays as described above. Both the wild-type and the m-FEZ1 clones were functional and were able to induce similar levels of resistance to both MLV-N-neo and HIV-1-puro infections (see Supplementary Fig. 1). These data suggest that mutation(s) elsewhere in the genome of the original R3-2 line resulted in overexpression of both alleles of FEZ1 in trans, conferring resistance to retroviral infection. 
Overexpression of FEZ1 blocks infection in human cells

To investigate whether overexpression of rat FEZ1 would similarly block HIV-1 infection in human cells, six clones of $293 \mathrm{~T}$ cells stably expressing variant mycHis-tagged FEZ1 (m-FEZ1), two control 293T cells containing empty vector, and wild-type $293 \mathrm{~T}$ cells were incubated with different amounts of recombinant HIV-1puro. As a positive control, the parental mutant line, R3-2, was also included in the infection assay. Overexpression of FEZ1 blocked HIV-1-puro infection in 293T cells compared with cells transduced with the empty vectors (Fig. 3A). As observed for the Rat2 stable lines (Fig. 2C), different levels of resistance were detected in

A
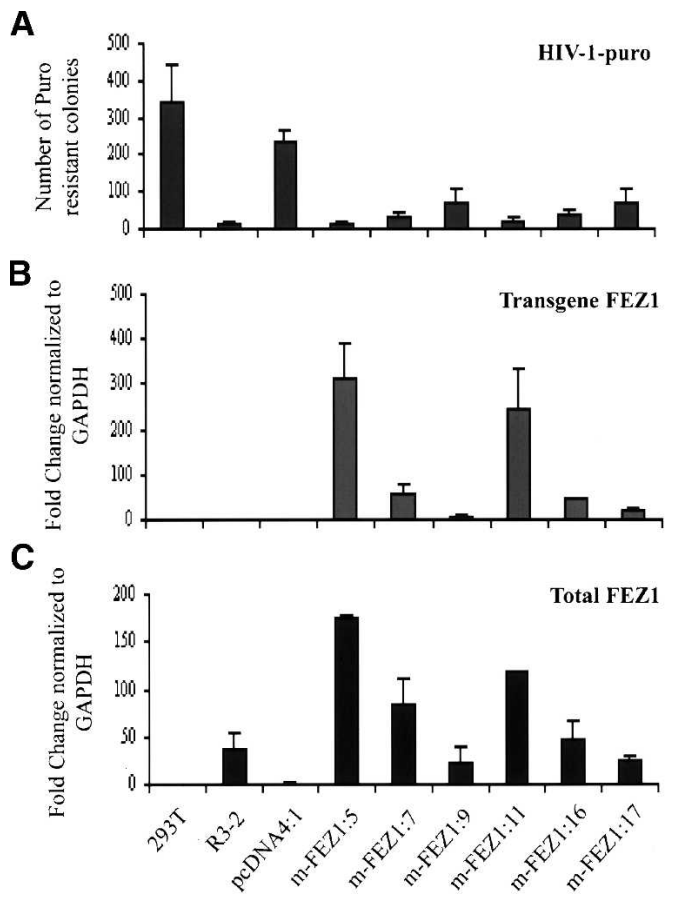

Figure 3. The pattern of expression levels of FEZ1 transcripts correlates with the levels of retroviral resistance in the various 293 T lines. (A) Overexpression of FEZ1 blocks retrovirus infection in $293 \mathrm{~T}$ cells. Cells transduced with the empty vector pcDNA4 or the pcDNA4 vector expressing FEZ1 originated from the mutant R3-2 line, m-FEZ1, were incubated with various amounts of HIV-1-puro. Cells were selected in medium containing puromycin and the number of the transduced colonies were then counted after $8-12 \mathrm{~d}$. The wild-type $293 \mathrm{~T}$ cells and the parental R3-2 line were included as negative and positive controls, respectively. Similar results were obtained in at least three independent experiments. $(B, C)$ Quantitative RT-PCR showing the level of FEZ1 expression in 293T cells. Cytoplasmic RNA was prepared from the same cell lines used in the transduction assay. The ds cDNA was generated and used as template in RT-PCR assays. Primers unique to each cDNA sequences were used to distinguished the transgene FEZ1 $(B)$ from the total (endogenous and transgene) FEZ1 transcripts $(C)$. The FC ratio between the $293 \mathrm{~T}$ mutant lines and the control empty vector line (pcDNA4:4) are given as median copy numbers normalized to GAPDH copy numbers. Two independent RNA preparations were used in the analysis. different 293T FEZ1-expressing lines, with the highest being 18-fold (m-FEZ1:5) in comparison with cells containing the empty vector (pcDNA4:4). Thus, overexpression of FEZ1 was sufficient to block HIV-1 in human 293 T cells.

\section{The level of FEZ1 expression in cultured cells correlates with virus resistance}

To determine whether the levels of retroviral resistance detected in the Rat2 and 293T FEZ1-expressing cells correlated with the expression pattern of FEZ1 in these lines, we used a quantitative real-time PCR assay. Primers unique to each cDNA sequences were used to distinguished the transgene FEZ1 from the total (endogenous and transgene) FEZ1 transcripts. The median FC ratios for FEZ1 expression between the FEZ1-expressing clones and the control empty vector lines in Rat2 and 293T cells are presented in Figures 2 and 3, respectively. In general, all the variant FEZ1 (m-FEZ1) stable lines tested showed similar pattern of expression levels for the transgene (Figs. 2D, 3B) and the total (Figs. 2E, 3C) FEZ1. Consistent with the data obtained from microarray analysis, $>30$-fold higher expression of the endogenous FEZ1 was detected in the mutant R3-2 in comparison with that in the wild-type Rat2 (Fig. 2E) or 293T cells (Fig. 3C). As expected, no transgene FEZ1 was expressed in either the wild-type, Rat2, or $293 \mathrm{~T}$ cells containing the empty pcDNA4 vectors) or R3-2 (Figs. 2D, 3B). Critically, the pattern of expression levels of FEZ1 transcripts correlated well with the levels of resistance to both MLV (Fig. 2A,B) and HIV-1 (Figs. 2C, 3A) infections in the various lines. Thus, the antiretroviral activity detected in the Rat 2 and $293 \mathrm{~T}$ stable lines is very likely attributable to the overexpression of FEZ1.

\section{Identification of the timing of the viral block in an FEZ1-expressing line}

The viral block in the R3-2 mutant line was previously shown to occur after reverse transcription but before nuclear entry (Gao and Goff 1999). To examine whether a similar block was occurring in the FEZ1-overexpressing lines, the course of viral DNA synthesis was examined after acute infection of the cells. One of the variant FEZ1-expressing clones (m-FEZ1:8) that blocked retroviral infection to almost the same extent as R3-2 and showed a similar level of transgene FEZ1 expression as the endogenous level of FEZ1 detected in R3-2 (Fig. 2E) was chosen for this purpose. The m-FEZ1:8 line, the control empty vector line pcDNA4:5, the wild-type Rat2 cells, and the parental R3-2 line were infected with ecotropic MLV-GFP (Gao and Goff 1999) at various dilutions. At different times after infection, total DNA was extracted, and different intermediates in the course of viral DNA synthesis were analyzed by RT-PCR. Total viral DNA could be detected $24 \mathrm{~h}$ after infection using primers specific for the GFP sequences, as described pre- 
viously (Gao and Goff 1999). The levels of the amplified viral DNA product correlated well with the levels of the input virus, demonstrating that the assay was not saturated but responded to input DNA (Fig. 4A). Comparable levels of viral DNA were detected in all the clones, except in the pcDNA4:5, which showed an increase at all dilutions of the input virus (Fig. 4A). These data confirmed the previous report (Gao and Goff 1999) and further indicated that, similar to R3-2, the FEZ1-expressing line had no defect in overall synthesis of viral DNA. We also examined the earlier step in reverse transcription by using primers that detect minus strand strong stop (MSS) DNA, the first detectable product of DNA synthesis. As expected, the levels of this DNA was also similar in the mutant lines (R3-2 and m-FEZ1:8) and the control cells (pcDNA4:5 and wild-type Rat2) (Fig. 4B) 24 h post-infection.

To test for the nuclear viral DNA forms, the levels of circular viral DNAs were analyzed using primers located

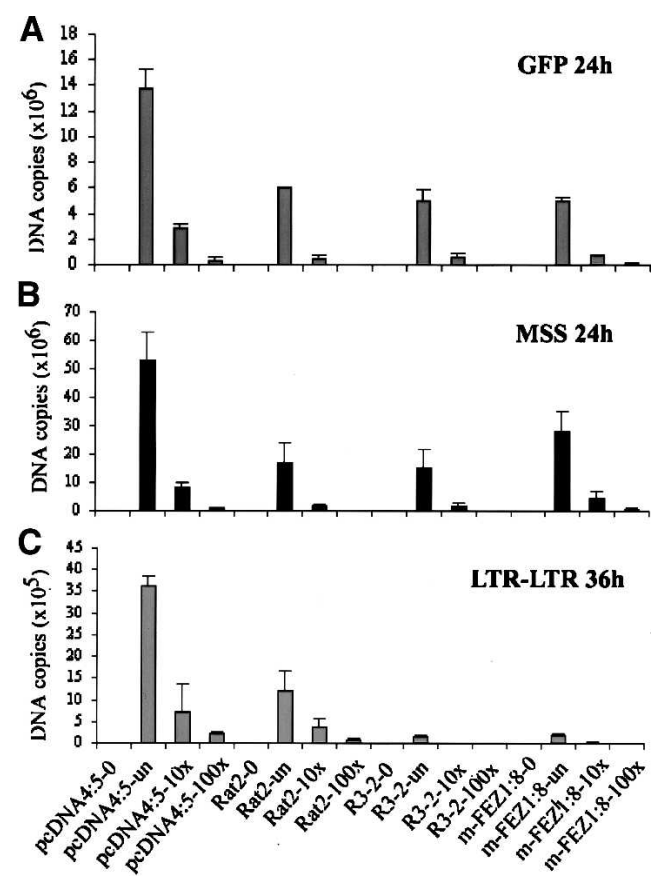

Figure 4. Quantitative RT-PCR indicating the localization of the viral block in FEZ1 stable line. One of the Rat2 line stably expressing FEZ1 (m-FEZ1:8), the control empty vector pcDNA4:5 line, the wild-type Rat2 cells, and the parental R3-2 were infected with either undiluted (un) or 10-fold serially diluted $(10 \times, 100 \times)$ ecotropic MLV-GFP. As a negative control, uninfected cells (indicated as 0 after the sample name) for each sample were included in the experiment. Total DNA was isolated at 24 and $36 \mathrm{~h}$ after infection and the amount of viral DNA synthesized in the infected cells was measured by RT-PCR. Using primers specific to GFP sequences, minus strand strong stop (MSS) DNA or LTR-LTR junction, the amounts of the total viral DNA $(A)$, MSS DNA $(B)$, or circular viral DNA in the nucleus $(C)$ were determined, respectively. The DNA copy number for each sample was normalized to the copy number of the internal control GAPDH. Each DNA sample was assayed in duplicates at the minimum of three different time points. in the U5 and U3 portions of the MLV long terminal repeat (LTR), as previously described (Gao and Goff 1999). High levels of circular DNA were found $36 \mathrm{~h}$ after infection in the control pcDNA4:5 line and Rat2 cells (Fig. 4C). Consistent with the previous report, very low levels of this DNA were observed in R3-2 cells (Gao and Goff 1999). Importantly, low levels were also seen in the $\mathrm{m}$-FEZ1:8 line, suggesting a similar block after reverse transcription and before nuclear entry in these lines. The amount of DNA was reduced 20- to 120-fold in both of these lines compared with that seen in the control pcDNA4:5 line. Similar results were obtained in an independent experiment using a low-molecular-weight DNA extract (Hirt 1967) as template in the RT-PCR assays described here (data not shown). These data indicate that overexpression of FEZ1 recreates the viral block observed in R3-2 cells and therefore suggest that overexpression of FEZ1 is likely the source of the viral resistance phenotype in this line.

\section{Knockdown of FEZ1 by RNA interference (RNAi) relieves the block to infection}

To assess the contribution of FEZ1 to the resistant phenotype of the mutant R3-2 line, RNAi was used to reduce FEZ1 levels in this line and the consequences were assessed by challenge of the cultures by infection with genetically marked retroviruses. Wild-type or mutant cells were treated with three independent predesigned short-interfering RNA duplexes (Ambion) or control duplexes. All the specific RNAi duplexes induced an approximately fourfold increase in susceptibility to MLV$\mathrm{N}$-neo infection, while a nonspecific siRNA duplex targeted to GFP had no effect (Fig. 5). As expected, wild-type Rat2 cells, which express low levels of FEZ1, were unchanged in their susceptibility to infection. The partial increase in susceptibility upon knockdown may reflect activity of RNAi in a subset of the cells, or general toxic effect of the transfection protocol. While the increase in susceptibility was not complete, the results strongly suggest that FEZ1 is required for the resistance phenotype of the mutant line.

FEZ1 does not increase the expression
of the other candidate resistance genes

The R3-2 line overexpressed a large number of genes, and though overexpression of FEZ1 was shown to be necessary and sufficient to induce resistance, it was possible that its mode of action involved the induction of some or all of the other differentially expressed genes originally identified in this study. To test whether the resistance phenotype of FEZ1-overexpressing lines required the FEZ1-induced expression of one or more of the other candidate resistance genes, quantitative RT-PCR was used to determine the levels of each of these genes in the FEZ1-expressing (m-FEZ1:8) line, the mutant R3-2 line, a line containing the empty vector (pcDNA4:5), and wildtype Rat 2 cells. The average fold increase in the expres- 


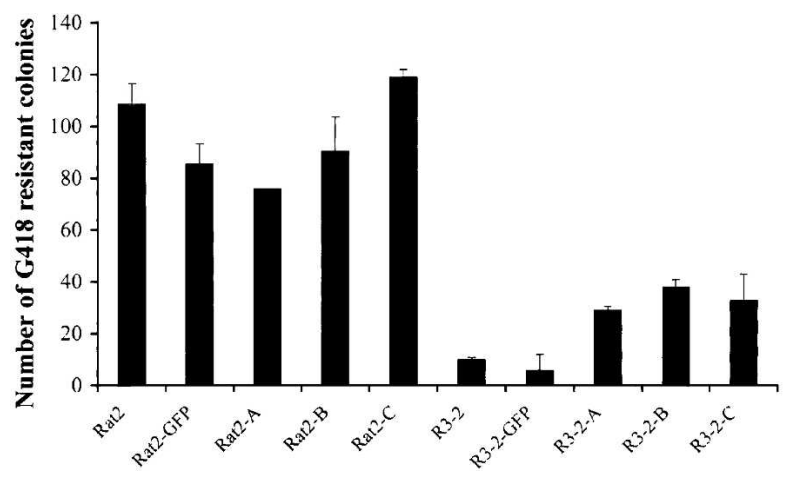

Figure 5. RNAi to FEZ1 reduces the resistance of the mutant R3-2 line. Wild-type or mutant R3-2 cells were transfected with three independent short-interfering RNAs (indicated as A, B, or C) on two consecutive days with equal amounts of RNA duplexes and subsequently seeded and infected with various amounts of MLV-N-neo. Cells were selected in medium containing G418, and the number of the transduced colonies was then counted after 8-12 d. The wild-type Rat2 cells and a nonspecific GFP duplex were included as negative controls. Similar results were obtained in at least three independent experiments.

sion of each of the candidate genes (except for the TALDH gene) in comparison with the wild-type Rat 2 cells is indicated in Table 2. The levels of expression for the five tested candidate genes in the FEZ1-expressing line were comparable to that of the control lines. Thus, FEZ1 did not affect the expression of any of the other candidate genes, suggesting that FEZ1 alone was sufficient to recreate the block in R3-2 cells and that overexpression of none of the other genes was necessary for resistance. FEZ1 showed $>30$-fold higher expression in the m-FEZ1: 8 line as detected earlier (Fig. 2E). Consistent with the data obtained in Figure 1, all of the tested genes were up-regulated in the mutant R3-2 line compared with the wild-type cells. These results suggest that the other overexpressed genes are not involved in the resistance.

\section{Discussion}

Identification of host factors that block retroviral infection is important to our understanding of the overall process of viral replication and the evolution of cellular restriction factors. In this study we have used microarray chip analysis followed by quantitative RT-PCR confirmation to identify candidate genes that could explain the virus-resistant phenotype of the mutant R3-2 cell line previously described (Gao and Goff 1999). The R3-2 cell line was generated by chemical mutagenesis of Rat2 fibroblasts and selection for resistance to retroviral infection. We identified a number of genes that were up-regulated in the mutant line compared with that of the wildtype cells, at least five of which were neuronal genes, suggesting that a neuronal regulator may have been affected by the mutagenesis. The most strongly induced genes identified were differentiation markers for rat mast cells, rat mast cell protease (RMCP)- 8 and RMCP10 (Lutzelschwab et al. 1997); glutamate receptor sub- unit (GluR-K3) (Nakanishi et al. 1990), involved in mediation of excitatory neurotransmission in the brain; mammalian enhancer-of-split- and hairy-related protein (Sharp-1) (Rossner et al. 1997), a transcriptional repressor protein involved in regulating neuronal differentiation; tumor-associated aldehyde dehydrogenase (T-ALDH) (Jones et al. 1988), which catalyzes the oxidation of aromatic aldehyde during rat hepatocarcinogenesis; skeletal Myosin Heavy Chain (Ogata et al. 1993), involved in cellular contraction and motility; and FEZ1 (Bloom and Horvitz 1997), a neuronal transport protein.

The virus-resistant R3-2 cells allowed entry of retroviruses pseudotyped with various envelope proteins (Gao and Goff 1999). Normal levels of linear viral DNA synthesis but a reduction in the formation of two-LTR circular DNA were detected, indicating a block after reverse transcription but before nuclear entry in these cells. In addition, the viral DNA was trapped in the cytoplasm in a form that could not be readily extracted under conditions that allowed the DNA recovery from the wild-type-infected cells. These results suggested that the factor(s) responsible for the block in the mutant cells was directly or indirectly associated with the cytoskeleton and somehow hindered the movement of viral DNA into the nucleus. In this scenario, while the importance of the other six genes identified remains to be examined, the cytoskeletal transport protein FEZ1 became of particular interest to explain the resistant phenotype of the mutant R3-2 line.

Rat FEZ1 and FEZ2 homologs, originally called Zygin I and II, were identified as synaptotagmin-binding proteins (Bloom and Horvitz 1997). In contrast to FEZ1, which is expressed exclusively in rat brain (Bloom and Horvitz 1997), FEZ2 is detected weakly but ubiquitously in all tissues (Fujita et al. 2004). FEZ1 is the mammalian homolog of C. elegans UNC-76 protein, which is necessary for axonal outgrowth in nematodes (Bloom and Horvitz 1997). Expression of human FEZ1 restores the defect

Table 2. Quantitative RT-PCR showing the levels of expression of candidate genes in an FEZ1-overexpressing line

\begin{tabular}{lrcc}
\hline Gene & R3-2 & m-FEZ1:8 & pcDNA4:5 \\
\hline RMCP-8 & 394.9 & 1.6 & 4.8 \\
RMCP-10 & 302.7 & 1.2 & 3.8 \\
GluR-K3 & 61.8 & 0.4 & 0.3 \\
FEZ1 & 45.6 & 46.3 & 1.2 \\
Sharp-1 & 38.0 & 0.4 & 0.6 \\
Myosin & 36.1 & 0.8 & 0.2 \\
\hline
\end{tabular}

Cytoplasmic RNA was prepared from the wild-type Rat2 cells, the parental R3-2 line, a FEZ1-expressing clone (m-FEZ1:8) generated from Rat 2 cells, and the control line containing empty vector (pcDNA4:5). The double-stranded cDNA was generated and used as template in RT-PCR assays. Primers specific to each gene's cDNA sequences were used to amplify the total amount of the transcript. The fold-change ratio between the mutant lines (R3-2 and m-FEZ:8) and the control wild-type Rat2 cells are given as median copy numbers normalized to GAPDH copy numbers for each gene. Two independent RNA preparations were used in the analysis. 
in unc-76 mutated nematodes, indicating that both proteins are evolutionarily conserved on a structural and functional basis. FEZ1 is also a cellular substrate of PKC $\zeta$ and the cytoplasmic translocation of FEZ1 upon PKC $\zeta$ activation appears to be required for neurite extension in rat pheochromocytoma PC12 cells (Wooten et al. 1997; Kuroda et al. 1999). A possible role for FEZ1, among other membrane-fusion proteins, has also been suggested in the regulation of salivary exocytosis through interaction with SNAREs in the rat parotid gland (Imai et al. 2001). In addition, the interaction between kinesin-I, a microtubule motor protein required for intracellular transport, and UNC-76 has recently been shown to contribute to the transport of synaptic vesicle precursors in the Drosophila nervous system (Gindhart et al. 2003), suggesting a possible role of FEZ1 in the regulation of kinesin-dependent transport. FEZ1 also plays a crucial role in neurite outgrowth by interacting with DISC1 (disrupted-in-schizophrenia 1) in association with the actin cytoskeleton (Miyoshi et al. 2003; Honda et al. 2004). FEZ1 therefore appears to interact with cytosketal networks to regulate their transport functions. Its up-regulation in the R3-2 cell line whose block in viral replication is at the level of viral DNA transport into the nucleus is intriguing as several viruses (for review, see Campbell and Hope 2003), including HIV-1 (McDonald et al. 2002), utilize microtubule-mediated transport to facilitate nuclear localization of the viral genome in an infected cell. Using RNAi, significant knockdown of FEZ1 expression in the mutant R3-2 line resulted in an increase in susceptibility to retroviral infection. To further test if FEZ1 could be responsible for the block in viral DNA transport in the resistant R3-2 cell line, FEZ1 was overexpressed in the parental Rat2 fibroblasts, as well as human 293T cells and tested for resistance to infection with genetically marked retroviruses. In cultured cells, overexpression of FEZ1 originating from either wild-type or R3-2 cells confers resistance to MLV and HIV-1 infection without affecting the expression of the other resistance candidate genes identified in this study. While in the mutant R3-2 line, these genes may be upstream of FEZ1 and play a role in its induction, resulting in the resistance phenotype of this line, or play additional as yet to be determined roles in viral resistance, FEZ1 alone was clearly sufficient to recreate the block to infection observed in R3-2 cells. Furthermore, the degree of retroviral resistance in these cells tightly correlates with the expression level of FEZ1. Finally, FEZ1-expressing rat cells show a similar phenotype to that of the mutant R3-2 line: Infection results in normal viral DNA synthesis but a reduction in the formation of circular DNA, indicating a block after reverse transcription but before nuclear entry. Therefore, overexpression of FEZ1 reproduces the block observed in R3-2 cells and may interfere with the process of viral DNA transport into the nucleus.

Recently, the retroviral resistance factor TRIM $5 \alpha$ (Stremlau et al. 2004) was shown to block infection soon after entry and prevents the activation of reverse transcription. The discovery that FEZ1 has the ability to block viral DNA transport into the nucleus suggests that certain host factors can impede various stages of the viral life cycle, albeit in a dose-dependent manner. Cell types expressing high levels of FEZ1 may be less susceptible to retroviral infection. Clearly FEZ1 differs from the classic restriction factors Fv1 and TRIM5 $\alpha$ in that it imposes a block to retroviral infection only when expressed at elevated levels. Of interest, the block imposed by FEZ1 bears a striking resemblance to that imposed by the dominant resistant murine gene Fv1 (Pincus et al. 1971), although the exact mechanism of its action remains unknown. Viral restriction factors and genes that can induce resistance such as FEZ1 operating at multiple points in the viral lifecycle would ensure multiple blocks to infection and suggests the possible existence of further inhibitory factors yet to be identified. Thus, identification and subsequent characterization of novel genes with antiviral activity should provide important information about host genes required for retroviral replication and suggest novel targets for therapeutic intervention.

\section{Materials and methods}

\section{Cell culture and RNA preparation}

Wild-type Rat2 cells and the mutant cell line R3-2 were maintained as described previously (Gao and Goff 1999). Total RNA was isolated from the trypsinized Rat2 cells and the mutant cell line by using QIAGEN's RNeasy total RNA isolation kit. mRNA was then isolated from the total RNA using the QIAGEN's Oligotex mRNA kit according to the manufacturer's protocols.

cRNA preparation for generation of gene expression profiles

Double-stranded (ds) cDNA was synthesized from $5 \mu \mathrm{g}$ of mRNA using Superscript ds cDNA synthesis kit from Invitrogen following the manufacturer's protocol. The ds cDNA was then purified using phase lock gels (PLG)-phenol/choloroform extraction and ethanol precipitation. Biotin-labeled cRNA was synthesized by an in vitro transcription reaction using the BioArray HighYield RNA transcript labeling kit (Enzo Diagnostics). cRNA was then purified using a RNeasy Mini kit (QIAGEN) according to the manufacturer's protocol and ethanol precipitated. Forty micrograms of the purified cRNA was fragmented according to the Affymetrix protocol and purified using G-25 sephadex columns (Roche). Fifteen micrograms of the cRNA probe was then hybridized to Rat2 Genome arrays (U34A) (Affymetrix, Inc.).

\section{Analysis of the gene expression profiles}

Experiments were performed in four replicates using total RNA prepared at two different time points from the wild-type or the R3-2 mutant cell line. To identify differentially expressed transcripts, the signal intensities for the experimental sample (mutant) were compared with the control sample (wild type) by software using the Global method of Scaling/normalization (Microarray Suite 5.0; Affymetrix, Inc.). Each of the two mutant samples was compared with the two wild-type samples for each time point resulting in eight pairwise comparisons for both samples. Quantitative changes in gene expression of all the 
8800 genes were calculated as signal $\log$ ratio (SLR) for each comparison. SLRs were signal intensities for each probe pair of the experimental and the control data set expressed in a logarithmic scale of base 2. SLRs were then calculated in multiples of two to obtain the FC for each transcript as described by Affymetrix. Only genes displaying a SLR $\geq 3$ (eightfold change in the expression level) and a detection $p$-value $<0.05$ were considered to be statistically significant. FC ratios were sorted by ascending or descending numbers to obtain the up-regulated and the down-regulated transcripts in R3-2 line, respectively.

\section{Quantitative real-time PCR}

The Affymetrix data for genes displaying a $\geq 16$-fold average change and a detection $p$-value $<0.05$ in the expression level across the whole panel were confirmed using quantitative realtime PCR with the TaqMan Model 7700 Sequence Detector (ABI-Perkin Elmer). Cytoplasmic RNA was isolated from the wild-type or the mutant cells $\left(2 \times 10^{6}\right)$ using QIAGEN's RNeasy total RNA isolation kit according to the standard protocol. Samples were treated twice with RNase-free DNase (Qiagen) while on the column before elution. Eight micrograms of RNA was converted into ds cDNA (see above), and $2 \mu \mathrm{L}$ of 1:10 dilution was utilized for 40 cycle (for $15 \mathrm{sec}$ at $94^{\circ} \mathrm{C}$; for $1 \mathrm{~min}$ at $60^{\circ} \mathrm{C}$, and for $1 \mathrm{~min}$ at $72^{\circ} \mathrm{C}$ ) three-step PCR using $25 \mu \mathrm{L}$ SYBR Green JumpStart Taq ReadyMix (Sigma) and $200 \mathrm{nM}$ of each primer according to the manufacturer's instructions. All reactions had a negative control in which no RT was added. Amplicons of 387-767 nucleotides were generated for each gene using primers corresponding to the specific 3'UTR region of each gene. For the primer sequences for amplification of each specific gene and the PCR product sizes, see Supplementary Table 1. Amplicon size and reaction specificity was confirmed by agarose gel electrophoresis. The number of target copies in each sample was interpolated from its detection threshold $\left(C_{T}\right)$ value using a GAPDH plasmid standard curve included in each plate. The plasmid containing the GAPDH amplicon was a kind gift from Dr. Luban (Department of Microbiology, Columbia University, New York). The PCR primers for mouse GAPDH were as follows: forward, 5'-CCTTCATTGACCTCAACTACATG G-3'; reverse, 5'-CGTGGTTCACACCCATCACAAAC-3'.

For detection of FEZ1 expression levels, cytoplasmic RNA was prepared from the parental R3-2 line, FEZ1-expressing clones generated from Rat 2 or $293 \mathrm{~T}$ cells, and control lines containing the empty vector as described above. The total and the transgene FEZ1 transcripts were distinguished using primers specific to FEZ1 sequences or one of the oligos corresponding to the sequences outside of the FEZ1 cloning site, respectively. The primer sequences for amplification of the transgene FEZ1 were as follows: pcDNA4-5, 5'-GGACCGATCCAGCCTCCG GACTCTAGCGTT-3' and mFEZ1-A5, 5'-CCAAACCTCCT CATCCCGAAG-3'. The primers mFEZ1-S3 (5'-CAGAATAA GCAGAAGGAGCAG-3') and m FEZ1-A3 (5'-GGTTGGACAG AGCACTTTTAA-3') were used for amplification of the total FEZ1 mRNA.

\section{Generation of FEZ1 expression vectors and stable lines}

Full-length Rat2 FEZ1 sequences, 1179 bp, was amplified using cDNA (see above) from the wild-type Rat2 fibroblasts or the mutant R3-2 line as templates. The PCR was performed by incubation for $1 \mathrm{~min}$ at $94^{\circ} \mathrm{C}, 1 \mathrm{~min}$ at $55^{\circ} \mathrm{C}$, and $2 \mathrm{~min}$ at $72^{\circ} \mathrm{C}$ for 35 cycles. The sequences of the primers for generation of $\mathrm{C}$ terminal myc-His-tagged FEZ1 were as follows: forward primer, FEZ1-S1-BamHI, 5'-GCTGATGGATCCACCATGGAGGCTC CACTGGTGAGT3'; reverse primer, FEZ1-A3-XhoI, 5'-GCA
ACGCTCGAGCAGGTTGGACAGAGCACTTTTAAA-3'. The sequences of the primers to produce C-terminal Flag-tagged FEZ1 were as follows: forward primer, FEZ1-S7-BamHI, 5'-GC TGATGGATCCGCCACCATGGAGGCTCACTGGTGAGT3'; reverse primer, FEZ1-A8-Flag-XhoI, 5'-GCAACGCTCGAG TTACTTGTCGTCATCGTCTTTGTAGTCGGTTGGACAGA GCACTTTTAA-3'. The restriction enzyme sites are underlined, and the Flag peptide sequence is in italics. The PCR products, wild-type FEZ1 (wt-FEZ1) or variant FEZ1 (m-FEZ1), were then cloned into a pcDNA4/TO/myc-HisB vector (Invitrogen), and the full-length inserts were confirmed by sequencing.

To generate cell lines stably overexpressing myc-His-, or Flagtagged FEZ1, $3 \times 10^{6}$ Rat2 fibroblast cells or $293 \mathrm{~T}$ cells were transfected with $4 \mu \mathrm{g}$ of the wild-type FEZ1 (pcDNA4-wt-FEZ1), the variant FEZ1 (pcDNA4-m-FEZ1), or the empty vector (pcDNA4) using FuGENE 6 transfection reagent (Roche). Cells were then subjected to selection in $50 \mu \mathrm{g} / \mathrm{mL}$ Zeocin $48 \mathrm{~h}$ after transfection until colony formation was observed. Six to 10 clones of each stable cell line (Rat2 or 293T) overexpressing either the wild-type (designated as wt-FEZ1 followed by the number of the clone) or the variant (designated as $\mathrm{m}-\mathrm{FEZ} 1$ followed by the number of the clone) FEZ1 and two clones of the empty vector lines (pcDNA4 followed by the number of the clone) were further tested in infection assays.

\section{Producer cell line and viruses}

ECO-Phoenix-MLV, the ecotropic phoenix producer cells (Kinsella and Nolan 1996) stably transfected with a green fluorescent protein (GFP) expression vector (Gao and Goff 1999) were used as the source of MLV-GFP virus. MLV (Towers et al. 2000) and HIV-1 (Naldini et al. 1996) viral vectors were prepared by transfection of $293 \mathrm{~T}$ cells using a combination of three expression vectors as described previously (Besnier et al. 2002). VSV-G expression vector (Naldini et al. 1996) was used to supply envelope for MLV and HIV-1 preparations. Throughout the text, viruses are named according to the origin of the Gag-Pol $(\mathrm{N}$ MLV, B-MLV, or HIV-1) and the reporter gene encoded within the packageable genome (either Neomycin or Puromycin). Thus, an HIV-1 particle carrying a puromycin resistance gene is referred to as HIV-1-puro, and a N-tropic or B-tropic MLV particle carrying a neo gene is referred to as MLV-N-neo or MLVB-neo, respectively.

\section{Infection of cultured cells and transduction assays}

Target cells $\left(1 \times 10^{5}\right)$ were seeded in either six-well plates or $10-\mathrm{cm}$ dishes $1 \mathrm{~d}$ before infection. All infections were performed in the presence of $8 \mu \mathrm{g} / \mathrm{mL}$ polybrene. Infections were carried out for $5 \mathrm{~h}$ or over the night to increase the infection efficiency. For virus titration of MLV-GFP, cells were inoculated with either undiluted or 10-fold serially diluted virus. Infected target cells were enumerated $48 \mathrm{~h}$ after infection by FACS analysis on a FacsCalibur instrument (Becton Dickinson) using CELLQUEST software as described (Bock et al. 2000). MLV-neo titer was measured by infection of Rat2 cells and colony counting after selection in G418 $(500 \mu \mathrm{g} / \mathrm{mL})$. HIV-1-puro titer was measured by infection of Rat 2 cells and colony counting after selection in $1.5 \mu \mathrm{g} / \mathrm{mL}$ Puromycin.

\section{Quantitative real-time PCR of viral DNA}

Cells were seeded in six-well plates $\left(1 \times 10^{5} /\right.$ well $) 1 \mathrm{~d}$ before infection. The wild-type or the mutant cell lines were infected with either undiluted or 10-fold serially diluted MLV-GFP virus. Total DNA was isolated at 24 or $36 \mathrm{~h}$ after infection using 
QIAGEN's DNeasy total DNA isolation kit according to the standard protocol. One microliter of the genomic DNA was utilized for $30 \mathrm{cycles}\left(30 \mathrm{sec}\right.$ at $94^{\circ} \mathrm{C}, 30 \mathrm{sec}$ at $55^{\circ} \mathrm{C}$, and $90 \mathrm{sec}$ at $72^{\circ} \mathrm{C}$ ) three-step PCR using $25 \mu \mathrm{L}$ of SYBR Green JumpStart Taq ReadyMix (Sigma), including $200 \mathrm{nM}$ of each primer as described above. Primers to amplify GFP sequence (GFP-FP and GFP-RP), the MLV minus strand strong stop DNA (MSS-FP and MSS-RP), and the MLV LTR-LTR junction, (MR5784 and MR4091) (Smith et al. 1997) have been previously reported (Gao and Goff 1999). Amplicon size and reaction specificity was confirmed by agarose gel electrophoresis. The number of target copies were calculated using a GAPDH plasmid standard curve as described above.

\section{RNAi}

Cells were seeded in six-well plates $1 \mathrm{~d}$ before transfection. Wild-type or mutant R3-2 cells $(30 \%-50 \%$ confluent) were transfected with three independent predesigned short-interfering RNAs (Ambion) using Oligofectamine transfection reagent (Invitrogen) according to the manufacture's protocol. A range of RNA duplexes between 150 and 600 pmol were tested. An siRNA duplex targeted to GFP was used as an nonspecific control. The sequences of GFP oligonucleotides were as follows: sense, 5'-rgrCUrArCrCUrgUUrCrCrAUrgrgrCrCrATT-3'; antisense, 5'-UrgrgrCrCrAUrgrgrArArCrArgrgUrArgrCTT-3'. Cells were transfected on two consecutive days with equal amounts of RNA duplexes and subsequently seeded, infected with various amount of MLV-N-neo, and selected as described above.

\section{Acknowledgments}

We thank Vladan Miljkovic at the Columbia Gene Chip Facility, Institute for Cancer Genetics DNA sequencing Facility, for his technical assistance in processing Affymetrix microarrays. We also thank Dr. Irene Nunes for valuable discussions and Dr. Greg Towers at the Wohl Virion Centre, Department of Immunology and Molecular Pathology, University College London, London, UK, for providing us with advice and retroviral vectors. M.H.N. is supported with a post-doctoral scholarship from The Swedish Foundation for International Cooperation in Research and Higher Education and The Swedish Research Council. M.H.N. and G.G. were Associates and S.P.G. is an Investigator of the Howard Hughes Medical Institute.

\section{References}

Besnier, C., Takeuchi, Y., and Towers, G. 2002. Restriction of lentivirus in monkeys. Proc. Natl. Acad. Sci. 99: 1192011925.

Bieniasz, P.D. 2003. Restriction factors: A defense against retroviral infection. Trends Microbiol. 11: 286-291.

Bloom, L. and Horvitz, H.R. 1997. The Caenorhabditis elegans gene unc-76 and its human homologs define a new gene family involved in axonal outgrowth and fasciculation. Proc. Natl. Acad. Sci. 94: 3414-3419.

Bock, M., Bishop, K.N., Towers, G., and Stoye, J.P. 2000. Use of a transient assay for studying the genetic determinants of Fv1 restriction. J. Virol. 74: 7422-7430.

Campbell, E.M. and Hope, T.J. 2003. Role of the cytoskeleton in nuclear import. Adv. Drug Deliv. Rev. 55: 761-771.

Fujita, T., Ikuta, J., Hamada, J., Okajima, T., Tatematsu, K., Tanizawa, K., and Kuroda, S. 2004. Identification of a tissue-non-specific homologue of axonal fasciculation and elongation protein $\zeta-1$. Biochem. Biophys. Res. Commun.
313: $738-744$.

Gao, G. and Goff, S.P. 1999. Somatic cell mutants resistant to retrovirus replication: Intracellular blocks during the early stages of infection. Mol. Biol. Cell 10: 1705-1717.

- 2004. Isolation of suppressor genes that restore retrovirus susceptibility to a virus-resistant cell line. Retrovirology 1: 30 .

Gao, G., Guo, X., and Goff, S.P. 2002. Inhibition of retroviral RNA production by ZAP, a CCCH-type zinc finger protein. Science 297: 1703-1706.

Gindhart, J.G., Chen, J., Faulkner, M., Gandhi, R., Doerner, K., Wisniewski, T., and Nandlestadt, A. 2003. The kinesin-associated protein UNC-76 is required for axonal transport in the Drosophila nervous system. Mol. Biol. Cell 14: 33563365.

Goff, S.P. 2004. Genetic control of retrovirus susceptibility in mammalian cells. Annu. Rev. Genet. 38: 61-85.

Harris, R.S., Bishop, K.N., Sheehy, A.M., Craig, H.M., PetersenMahrt, S.K., Watt, I.N., Neuberger, M.S., and Malim. M.H. 2003. DNA deamination mediates innate immunity to retroviral infection. Cell 113: 803-809.

Hatziioannou, T., Perez-Caballero, D., Yang, A., Cowan, S., and Bieniasz, P.D. 2004. Retrovirus resistance factors Ref1 and Lv1 are species-specific variants of TRIM5 $\alpha$. Proc. Nat1. Acad. Sci. 101: 10774-10779.

Hirt, B. 1967. Selective extraction of polyoma DNA from infected mouse cell cultures. J. Mol. Biol. 26: 365-369.

Honda, A., Miyoshi, K., Baba, K., Taniguchi, M., Koyama, Y., Kuroda, S., Katayama, T., and Tohyama, M. 2004. Expression of fasciculation and elongation protein $\zeta-1$ (FEZ1) in the developing rat brain. Brain Res. Mol. Brain Res. 122: 89-92.

Imai, A., Nashida, T., and Shimomura, H. 2001. mRNA expression of membrane-fusion-related proteins in rat parotid gland. Arch. Oral. Biol. 46: 955-962.

Jones Jr., D.E., Brennan, M.D., Hempel, J., and Lindahl, R. 1988. Cloning and complete nucleotide sequence of a full-length cDNA encoding a catalytically functional tumor-associated aldehyde dehydrogenase. Proc. Nat1. Acad. Sci. 85: 17821786.

Keckesova, Z., Ylinen, L.M., and Towers, G.J. 2004. The human and African green monkey TRIM $5 \alpha$ genes encode Ref 1 and Lv1 retroviral restriction factor activities. Proc. Natl. Acad. Sci. 101: 10780-10785.

Kinsella, T.M. and Nolan, G.P. 1996. Episomal vectors rapidly and stably produce high-titer recombinant retrovirus. Hum. Gene Ther. 7: 1405-1413.

Kuroda, S., Nakagawa, N., Tokunaga, C., Tatematsu, K., and Tanizawa, K. 1999. Mammalian homologue of the Caenorhabditis elegans UNC-76 protein involved in axonal outgrowth is a protein kinase $\mathrm{C} \zeta$-interacting protein. J. Cell Biol. 144: 403-411.

Lutzelschwab, C., Pejler, G., Aveskogh, M., and Hellman, L. 1997. Secretory granule proteases in rat mast cells: Cloning of 10 different serine proteases and a carboxypeptidase A from various rat mast cell populations. J. Exp. Med. 185: 1329.

McDonald, D., Vodicka, M.A., Lucero, G., Svitkina, T.M., Borisy, G.G., Emerman, M., and Hope, T.J. 2002. Visualization of the intracellular behavior of HIV in living cells. $J$. Cell. Biol. 159: 441-452.

Miyoshi, K., Honda, A., Baba, K., Taniguchi, M., Oono, K., Fujita, T., Kuroda, S., Katayama, T., and Tohyama, M. 2003. Disrupted-In-Schizophrenia 1, a candidate gene for schizophrenia, participates in neurite outgrowth. Mol. Psychiatry 8: 685-694.

Nakanishi, N., Shneider, N.A., and Axel, R. 1990. A family of 
glutamate receptor genes: Evidence for the formation of heteromultimeric receptors with distinct channel properties. Neuron 5: 569-581.

Naldini, L., Blomer, U., Gallay, P., Ory, D., Mulligan, R., Gage, F.H., Verma, I.M., and Trono, D. 1996. In vivo gene delivery and stable transduction of nondividing cells by a lentiviral vector. Science 272: 263-267.

Ogata, I., Saez, C.G., Greenwel, P., Ponce Mde, L., Geerts, A., Leinwand, L.A., and Rojkind, M. 1993. Rat liver fat-storing cell lines express sarcomeric myosin heavy chain mRNA and protein. Cell Motil. Cytoskeleton 26: 125-132.

Pincus, T., Rowe, W.P., and Lilly, F. 1971. A major genetic locus affecting resistance to infection with murine leukemia viruses, II: Apparent identity to a major locus described for resistance to friend murine leukemia virus. J. Exp. Med. 133: 1234-1241.

Rossner, M.J., Dorr, J., Gass, P., Schwab, M.H., and Nave, K.A. 1997. SHARPs: Mammalian enhancer-of-split- and hairy-related proteins coupled to neuronal stimulation. Mol. Cell. Neurosci. 9: 460-475.

Smith, C.M., Potts III, W.B., Smith, J.S., and Roth, M.J. 1997. RNase $\mathrm{H}$ cleavage of tRNAPro mediated by M-MuLV and HIV-1 reverse transcriptases. Virology 229: 437-446.

Stremlau, M., Owens, C.M., Perron, M.J., Kiessling, M., Autissier, P., and Sodroski, J. 2004. The cytoplasmic body component TRIM5 $\alpha$ restricts HIV-1 infection in Old World monkeys. Nature 427: 848-853.

Towers, G., Bock, M., Martin, S., Takeuchi, Y., Stoye, J.P., and Danos, O. 2000. A conserved mechanism of retrovirus restriction in mammals. Proc. Natl. Acad. Sci. 97: 1229512299.

Wooten, M.W., Zhou, G., Wooten, M.C., and Seibenhener, M.L. 1997. Transport of protein kinase $C$ isoforms to the nucleus of PC12 cells by nerve growth factor: Association of atypical

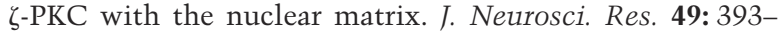
403. 


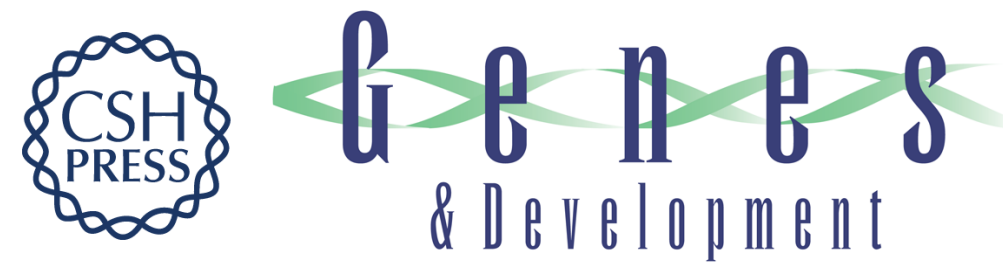

\section{Overexpression of fasciculation and elongation protein $\zeta-1$ (FEZ1) induces a post-entry block to retroviruses in cultured cells}

Mojgan H. Naghavi, Theodora Hatziioannou, Guangxia Gao, et al.

Genes Dev. 2005, 19:

Access the most recent version at doi:10.1101/gad.1290005

Supplemental http://genesdev.cshlp.org/content/suppl/2005/04/15/19.9.1105.DC1
Material

References This article cites 32 articles, 15 of which can be accessed free at:

http://genesdev.cshlp.org/content/19/9/1105.full.html\#ref-list-1

License

Email Alerting Receive free email alerts when new articles cite this article - sign up in the box at the top

Service right corner of the article or click here.

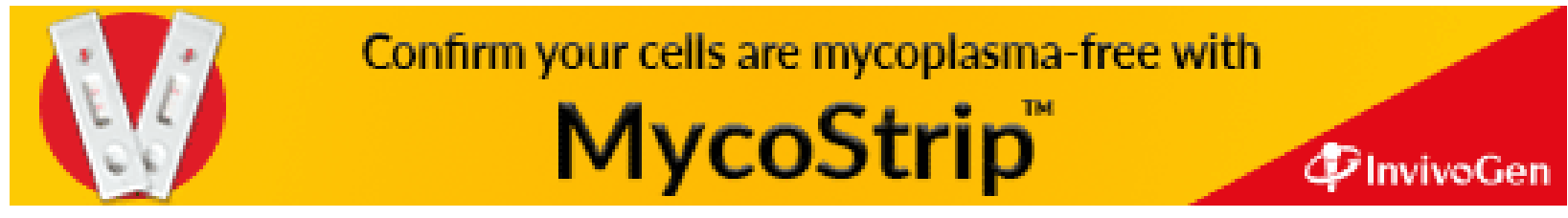

